\title{
ÖGUM News and Facts
}

\section{Laudatio für Gebhard Mathis}

Bei der Generalversammlung der ÖGUM am Dreiländertreffen in Leipzig 2019 wurde nach einstimmigem Beschluss am DLT in Basel 2018 - Herrn Prim. Univ. Prof. Dr. Gebhard Mathis die Ehrenmitgliedschaft der ÖGUM verliehen. Diese wurde vom amtierenden Präsidenten, Prof. Dr. H. Kathrein, im Namen der ÖGUM überreicht.

Gebhard Mathis kam 1950 in Feldkirch zur Welt, studierte Medizin in Wien und promovierte dort 1977. Seine Ausbildung zum Facharzt für Innere Medizin erfolgte 19771987 in verschiedenen Institutionen (KH Hohenems, LKH Feldkirch, AKH Wien, Medizinische Klinik St. Gallen). Der Facharzttitel wurde ihm 1987 zugesprochen. Von 1993-2006 leitete und formte er die Interne Abteilung am KH Hohenems. G. Mathis habilitierte 1994 an der Universität Innsbruck und erhielt den Titel Universitätsprofessor 1998. Seit 2006 ist er niedergelassener Facharzt für Innere Medizin in Rankweil.

Gebhard Mathis beschäftigte sich seit Beginn seiner Ausbildung mit abdominellem Ultraschall, fand aber sehr früh einen innovativen Zugang zur Thoraxsonografie, der über das Erkennen eines Pleuraergusses hinausging. Erste Publikationen zur Sonografie bei Lungenembolie erschienen 1990 in der Zeitschrift „Ultraschall in der Medizin“. Bemerkenswert erscheint, dass die Grundlagen dazu in einem kleinen, peripheren Krankenhaus erarbeitet wurden. Über Jahre gehörte Mathis dann zu einer Gruppe von Ärzten und Ärztinnen, die den Stellenwert der Thoraxsonografie entscheidend veränderten. Im letzten Jahrzehnt widmete sich Mathis sehr aktiv der Notfallsonografie in klinischer Forschung und Lehre.
Das publikatorische Werk des Geehrten ist eindrucksvoll. Gebhard Mathis kann 130 zitierbare Arbeiten (75\% davon als Erstautor) in angesehenen Zeitschriften (z. B. Ultraschall in Med, Chest, Eur Heart J, Eur J Rad, Praxis) vorweisen. Er ist Herausgeber und Autor mehrerer Lehrbücher und Autor vieler Buchbeiträge. „Lungen- und Pleurasonografie“, erschienen erstmals im Springer-Verlag 1992, muss man als „Klassiker“ bezeichnen und liegt nun in der 6 . Ausgabe vor.

Parallel dazu widmete sich Gebhard Mathis überaus engagiert der Aus- und Fortbildung der Kollegenschaft durch eine rege Vortrags- und Seminartätigkeit mit ca. 10 Kursen pro Jahr. Er ist schon lange ein geschätzter Redner bei in- und ausländischen Kongressen. Legendär sind die von ihm bis 2017 organisierten „Ultraschalltage“ in Gaschurn, bei denen in sehr amikaler Atmosphäre Wissen auf hohem Niveau vermittelt wurde.

Gebhard Mathis ist schon Jahrzehnte Mitglied der ÖGUM. Er war ab dem Jahr 2002 im Vorstand und von 2006-2010 engagierter und aktiver Präsident der Gesellschaft, der den weiten Weg von Vorarlberg nach Wien nie scheute. Im Jahr 1990 organisierte er als Kongresspräsident das 14. DLT in Bregenz. Von 2007-2013 bekleidete er eine Funktion im Education \& Professional Standards Committee der EFSUMB. Die europäische Gesellschaft nahm ihn 2017 als Fellow auf. Als ÖGUM-Präsident war er maßgeblich an der Entwicklung der Zertifikatsrichtlinie „Sonografie“ der Österreichischen Ärztekammer beteiligt, die einerseits für die Qualität der Ausbildung bürgt, und andererseits der niedergelassenen Kollegenschaft erst die Verrechnung von sonografischen Untersuchungen ermöglichte.

Eine Würdigung der Verdienste wäre unvollständig, wenn man seine Leistungen jenseits der Ultraschalldiagnostik nicht erwähnen würde. So war er seit 2007 Mitinitiator im Vorarlberger Vorsorge-Koloskopie-Programm und in der Sektion Vorarlberg der Österreichischen Krebshilfe, deren Präsidentschaft er seit 2012 innehat. In seine aktive Zeit im KH Hohenems fielen der Aufbau der Vorarlberger Palliativstation und der Aufbau eines Palliativlehrgangs in Vorarlberg. Für diese Tätigkeiten erhielt er hohe Auszeichnungen wie den Durig-Böhler-Preis, den Dr.-Toni-Russ-Preis sowie das große Verdienstzeichen des Landes Vorarlberg.

Die ÖGUM-Ehrenmitgliedschaft gilt zurecht einem empathischen Arzt und hervorragenden klinischen Wissenschaftler mit ungebrochenem Hang zu Innovationen, einem hoch engagierten klinischen Lehrer mit einem offenen Ohr für jüngere Kollegen und einem erfolgreichen Organisator von überaus geschätzten Fortbildungsveranstaltungen. Gebhard Mathis war immer ein Arzt, der für die ihm anvertrauten Kranken die gesamtheitliche Sicht einer menschlichen Medizin auf höchstem wissenschaftlichem Niveau pflegte. Die Ovationen in der Generalversammlung 2019 haben daher niemanden überrascht.

Hermann Kathrein und Hannes Gruber für die ÖGUM 